\title{
High energy properties of a sample of Neutron Star X-ray binaries with INTEGRAL*
}

\author{
Mariateresa Fiocchi \\ Istituto di Astrofisica Spaziale e Fisica Cosmica di Roma (INAF). Via Fosso del Cavaliere 100, \\ Roma, I-00133, Italy \\ E-mail: mariateresa.fiocchi@iasf-roma.inaf.it
}

\section{Pietro Ubertini ${ }^{\dagger}$}

Istituto di Astrofisica Spaziale e Fisica Cosmica di Roma (INAF). Via Fosso del Cavaliere 100, Roma, I-00133, Italy

\section{Angela Bazzano}

Istituto di Astrofisica Spaziale e Fisica Cosmica di Roma (INAF). Via Fosso del Cavaliere 100, Roma, I-00133, Italy

\section{Memmo Federici}

Istituto di Astrofisica Spaziale e Fisica Cosmica di Roma (INAF). Via Fosso del Cavaliere 100, Roma, I-00133, Italy

\begin{abstract}
Broad band observations are a powerful tool to study the accretion flows around compact objects. Recently significant progress have been possible because of INTEGRAL which has both broad band coverage and high sensitivity in hard X-ray. IBIS instrument observed a large fraction of the of known Low Mass X-Ray Binaries ( 43\%), allowing us to deeply investigate the spectral and timing properties of both persistent and transient sources. We report on some examples of bursters in typical low/hard or high/soft states, very spectacular transitions between two spectral states and detections of hard tail in the high energies spectra. Finally, we report on the average spectral characteristics of the ultra compact X-ray binaries in a broad band energy, using INTEGRAL, BeppoSAX and SWIFT data.
\end{abstract}

7th INTEGRAL Workshop

September 8-11 2008

Copenhagen, Denmark

*INTEGRAL is an ESA project with instruments and science data center funded by ESA member states (especially the PI countries: Denmark, France, Germany, Italy, Switzerland, Spain), Czech Republic and Poland, and with the participation of Russia and the USA.

† Speaker. 


\section{Introduction}

Low Mass X-ray Binaries (LMXB) are systems containing a late type (F,G,K) low mass donor star $\left(\mathrm{M} \sim 1 \mathrm{M}_{\text {solar }}\right)$ and a compact object with weak magnetic field $\left(10^{9-11} \mathrm{G}\right)$ that accrete mass via Roche lobe overflow. Generally, most of them are localized in the Galactic Bulge and can be characterized by type I X-ray bursts, a thermonuclear phenomena on the neutron star surface (burster).

The study of galactic X-ray binaries has maturated very rapidly in the past few year and has produced several exciting discoveries. RXTE has observed phenomena that take place close to the compact object, XMM-Newton and Chandra are producing energy spectra and image of unprecedent quality for some of object, radio observatory are beginning to show that jets are quite common in the X-ray binaries. The analysis of this data has posed new and more detailed questions which are again open problems. INTEGRAL instruments allowed us to study the hard X-ray emission of these sources, highlighting that most of them show spectra above about $100 \mathrm{keV}$.

The recent catalog of known LMXBs (Liu et al. 2007) contains 187 sources with $\sim 90$ bursters. Compared with the previous edition (Liu et al. 2001) the number of LMXBs in the Galaxy has increased by $\sim 30 \%$. The IBIS/INTEGRAL catalog Bird et al. (2007), reported 421 sources in the 20-100 keV energy range and among this source list we find 79 LMXBs, with a sky distribution shown in figure 1, of which 45 are Bursters. At very high energies $(>100 \mathrm{keV}) 26 \mathrm{LMXBs}$ have been detected, corresponding to $\sim 50 \%$ of the total sample (Bazzano et al. 2006).

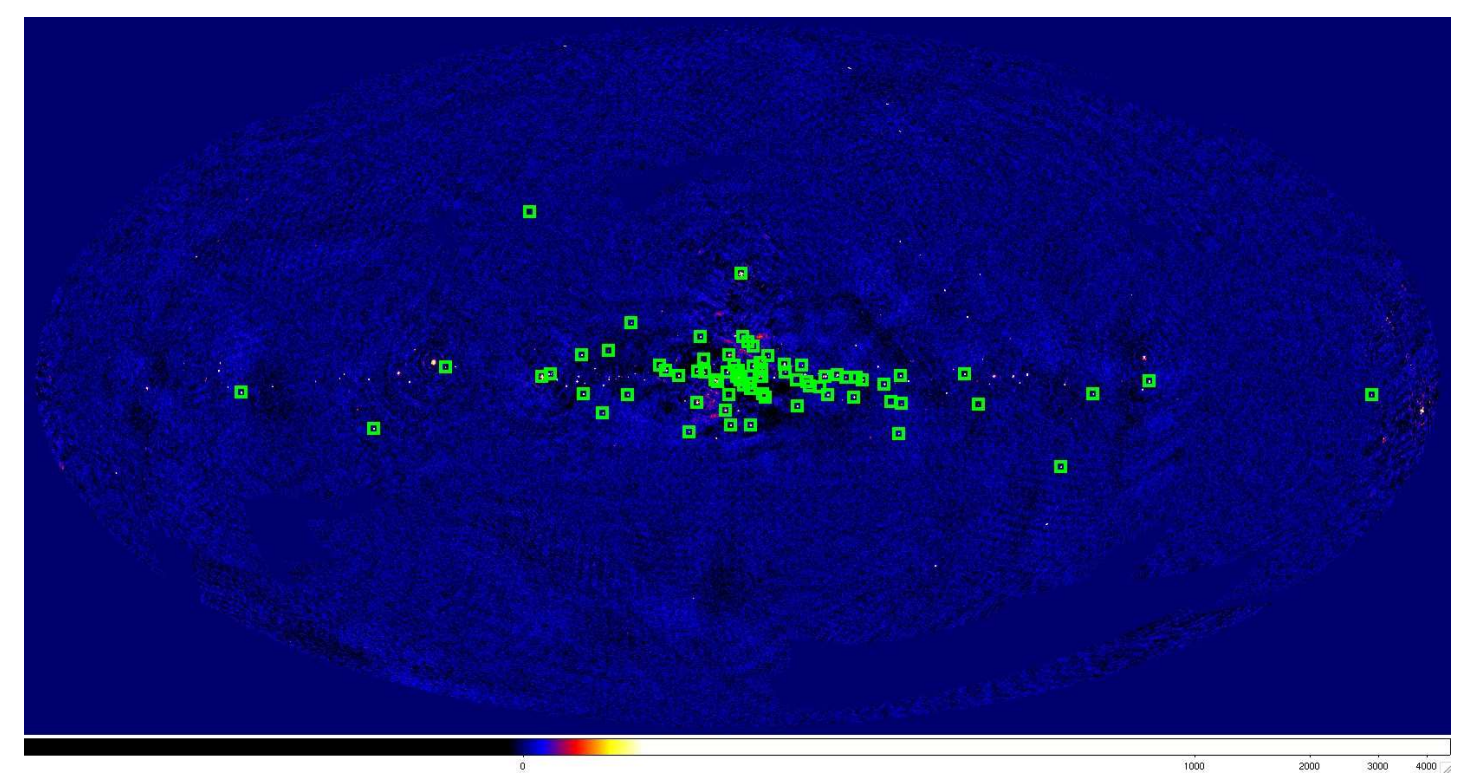

Figure 1: The 20-100 keV IBIS map with LMXBs location from Bird et al. 2007.

Today INTEGRAL instruments offer us an unique opportunity to study the high energy emission of LMXBs. Detailed analysis of these data helped us to understand the physical processes 
responsible for the observed emission. X-ray spectra of LMXBs are usually fit with a complex model: at low energies a black body component that approximates the spectrum of an optically thick, geometrically thin accretion disk and/or the neutron star surface, and at higher energies a Comptonization component due to repeated inverse Compton scattering of the soft seed photons by hot electrons plasma with a thermal distribution of velocities. IBIS/INTEGRAL spectra showed a more complex picture, detecting sources with a non thermal emission at high energies $(>50-60$ $\mathrm{keV}$ ) or allowing to study the temporal and spectral behavior of transient source. We report here some typical examples of LMXBs observed with INTEGRAL: persistent sources in low/hard state or in high/soft state, persistent sources showing standard spectral transition from a low/hard to high/soft state, transient sources during outburst and sources with spectra without any high energies cutoff. Finally, we describe the average spectral characteristics of the ultra compact LMXBs observed by IBIS/INTEGRAL.

\section{IGRJ 17597-2201 observed in a typical low/hard state}

This source is a persistent burster spending most of the time in a typical low hard state. Its spectrum, obtained using PN/XMM Newton and IBIS data in a very broad band (0.6-200 keV), is shown in figure 2. IBIS spectrum was generated in standard way using OSA6 software, with exposure time of $55 \mathrm{ks}$ and start time 53084.6 MJD. EPIC spectrum was generated using SAS software with exposure time of $879 \mathrm{~s}$ and start time 53085. It is well fitted with a single Comptonized component with electron temperature of $\sim 34 \mathrm{keV}$, the optical depth of $\sim 1.7$ and seed photon temperature of $\sim 0.7 \mathrm{keV}$, absorbed by a $\mathrm{N}_{H} \sim 1.6 \times 10^{22} \mathrm{~cm}^{-2}$. The fit gives a good $\chi^{2}\left(\chi^{2}=760 / 647\right)$.

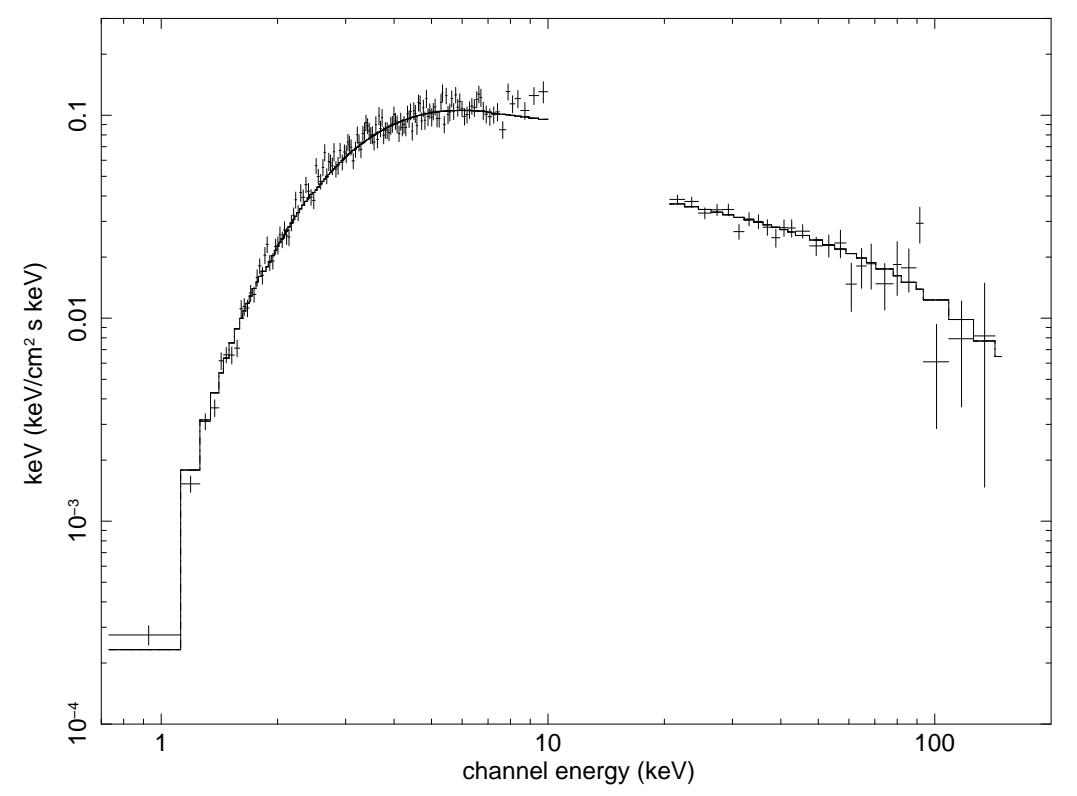

Figure 2: PN/XMM Newton and IBIS/INTEGRAL spectrum of the burster IGRJ 17597-2201 


\section{3. $4 \mathrm{U}$ 1705-44 during a typical spectral transition from an high/soft to low/hard state}

4U1705-440 is a binary system classified as an atoll sources, showing the variability on all time scales, from months down to milliseconds. It was observed four times in two different spectral state, using INTEGRAL and BeppoSAX data: during two ephocs the source was in the standard high/soft state and during other two ephocs it was in an low/hard state.

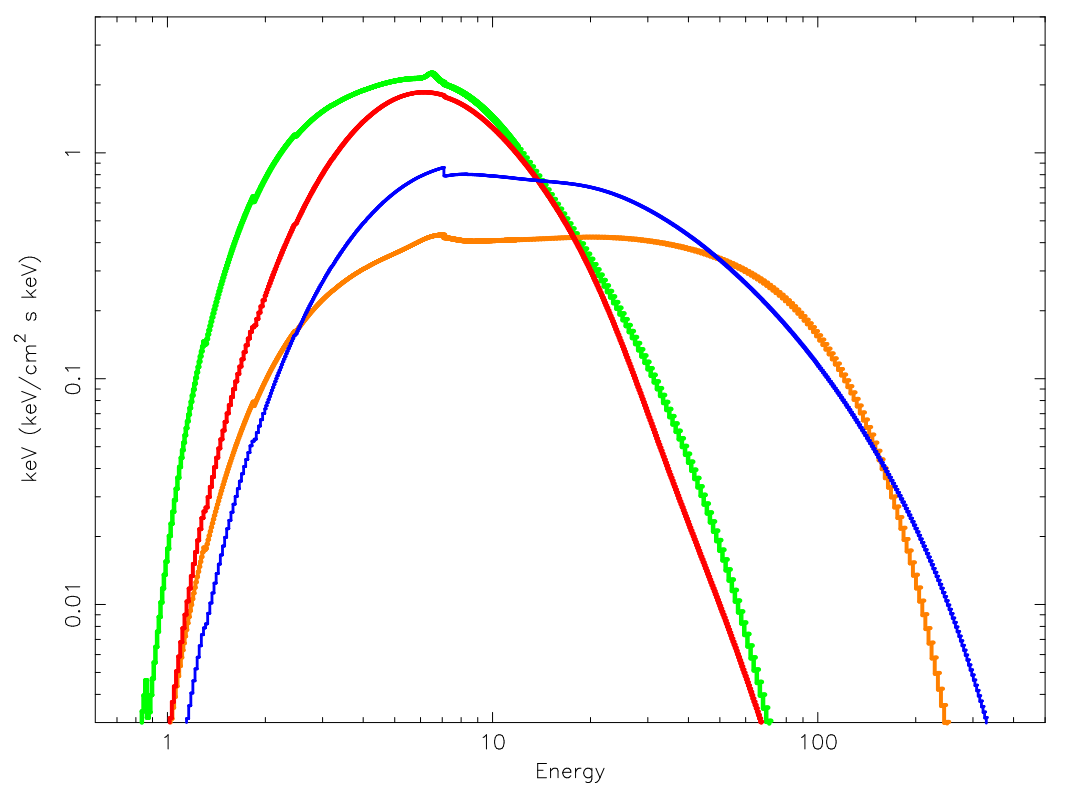

Figure 3: Comparison of the models used to fit data for the four observations of $4 \mathrm{U}$ 1705-44. BeppoSAX and INTEGRAL soft state, BeppoSAX and INTEGRAL hard state are showed in green, red, orange and blue respectively.

Details on data reduction and spectral fit results can be found in Fiocchi et al. 2007. Its energy spectrum in both states can be described as the sum of two blackbody, a 6.4-keV Fe line and a component due to thermal Comptonization, with a strong signature of Compton reflection in the INTEGRAL hard state (for details, see Fiocchi et al. 2007). These spectra are shown in figure 3, using the green and red color for INTEGRAL and BeppoSAX high/soft state, and the orange and blue color for INTEGRAL and BeppoSAX hard/low state, respectively. The blackbody components probably originate in the disk and on the neutron-star surface while the Comptonized component could arise from a hot inner flow with the seed photons originating from the disk and/or the neutron-star surface. These spectral transitions can be associated with variations in the accretion rate, changing in turn the temperature of the Comptonizing electrons and the strength of Compton reflection. These changes can be explained in a model with a truncated outer disk surrounding a hot inner flow, and the accretion rate determining the truncation radius.

We note that the presence of all three components (two black body and a comptonized component) can only be seen directly around the transition, where the disc has high enough temperature to contribute to the X-ray bandpass, while the boundary layer is not yet completely optically thick so 
as to Comptonized all the newtron star surface photons (Fiocchi et al. 2007).

\section{4U 1636-53: a burster with an hard tail at high energy}

$4 \mathrm{U} 1636-53$ is a binary system classified as an atoll sources and its spectral characteristics have been studied extensively only at low energy. With INTEGRAL and BeppoSAX we have studied its spectral behavior at high energies $(>20 \mathrm{keV})$, deeply investigating three different epochs corresponding to three different spectral states. Details on data reduction and spectral fit results can be found in Fiocchi et al. 2006. Two spectra show a continuum well described by one or two blackbody at low energies and Comptonized components. Changes in the Comptonizing electrons and black body temperature and accretion rates are typical of the spectral transitions from high/soft state to low/hard state (see Fiocchi et al. 2006 for details).

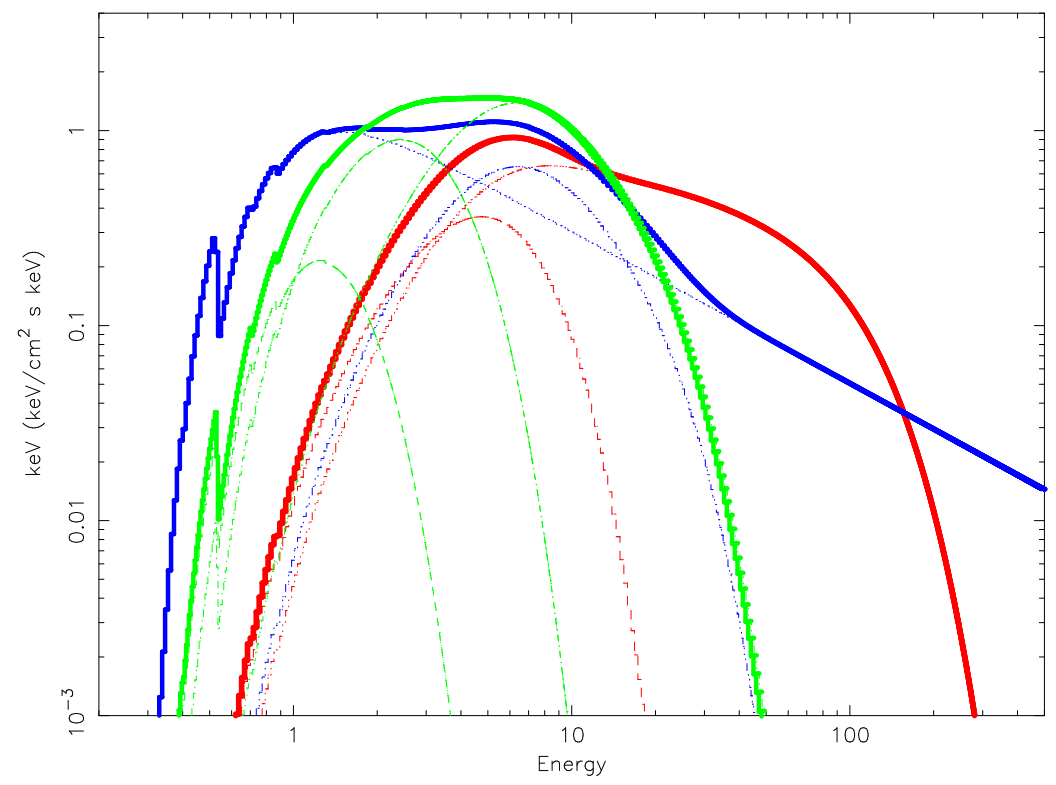

Figure 4: Comparison of the models used to fit data for the three observations of $4 \mathrm{U}$ 1636-53. BeppoSAX soft state, INTEGRAL hard state and a peculiar INTEGRAL state are showed in green, red and blue respectively.

In one occasion INTEGRAL spectrum shows, for first time in this source, a hard tail dominating the emission above $30 \mathrm{keV}$ (Figure 4, lines blue). This spectrum can be fitted as the sum of a Comptonized component with spectral parameters typicaly of a soft state and a overlapped power-law component $(\Gamma=2.76)$, indicating the presence of a non thermal electron distribution of velocities. A similar feature was observed in other LMXBs (GX 17+2, Di Salvo et al. 2000; GX 349+2, Di Salvo et al. 2001; GX 5-1, Asai et al. 1994; Sco X-1, D'Amico et al. 2001; 4U 1608522, Zhang et al. 1996; XB 1254-690 Iaria et al. 2001; Cir X-1, Iaria et al. 2002; 4U 0614+091, Piraino et al. 1999). A two-component electron distribution could arise from non-thermal electron acceleration regions powered by magnetic reconnections above a disc. Low-energy electrons 
cool preferentially by Coulomb collisions leading to a thermal distribution while the high-energy electrons cool by Compton scattering, preserving a non-thermal distribution (Coppi 1999). Alternatively, the thermal and non-thermal electrons could be spatially distinct, e.g. magnetic reconnection above the disc can produce a non-thermal electron distribution, while overheating of the inner disc produces the thermal Comptonization (Kubota et al. 2001). Other possibility is thermal and non thermal emission originates from the disc-jet coupling as also reported for GX 354-0 (Migliari et al. 2003).

\section{SAX J1810-269: a typical transient source}

The transient X-ray source SAX J1810.8-2609 was discovered on 1998 with the Wide Field Cameras on board the BeppoSAX satellite (Ubertini et al. 1998) and it remained in a quiescent state for most of the time. Only in August 2007 a new X-ray activity was observed. Figure 5 shows the RXTE/ASM and INTEGRAL/IBIS light curve of this recent outburst.
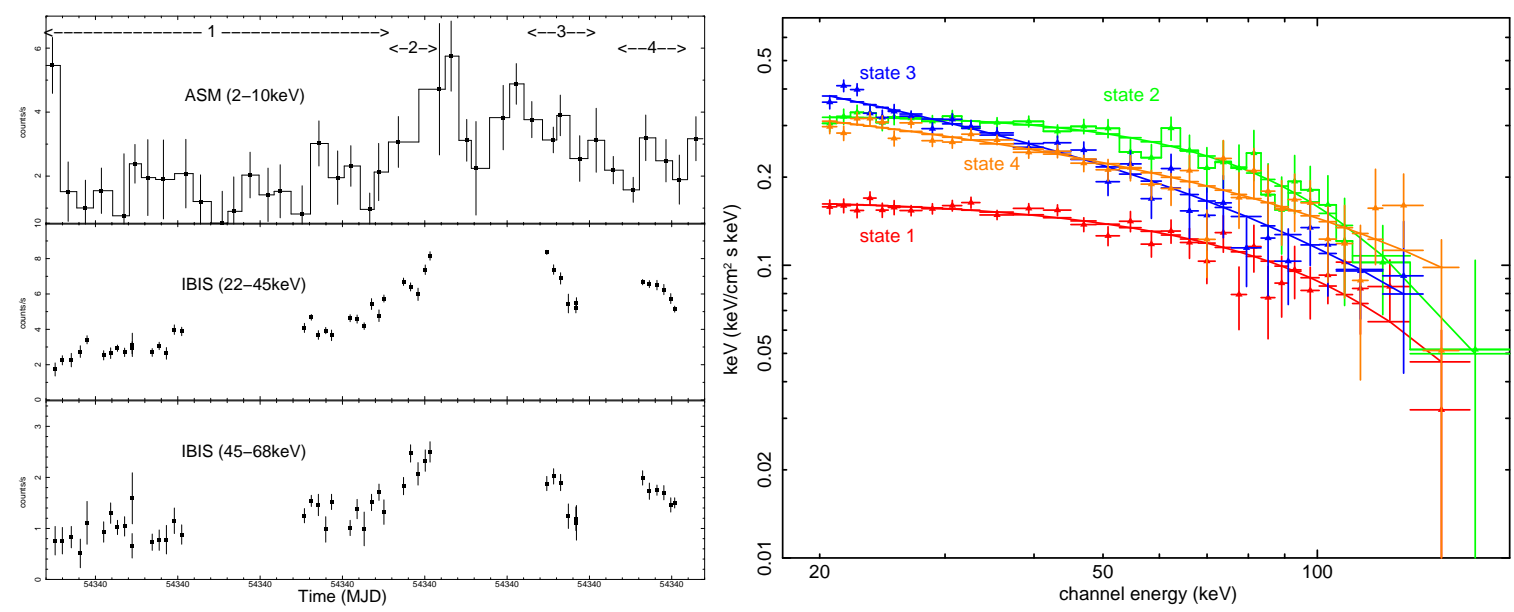

Figure 5: Left: a)The RXTE ASM light curve daily averaged in 2-10 keV energy band of SAX J1810.82609. b) The IBIS light curve in 22-45 keV energy band c) The IBIS light curve in 45-68 keV energy band. Right: Four different spectral state with the best models using COMPTT component

The IBIS observations has allow us to follow the high energy behavior of SAX J1810-269 during its long and bright outburst. Details on data reduction and spectral fit results can be found in Fiocchi et al. 2009. Spectra are well fitted with a simple power law after the outburst peak, while a COMPTт (or cutoff power law) model is required before the outburst peak. Figure 5 (right) show spectra in four epochs as indicated in the light curve (left panel). During the increase of the flux, the SAX J1810-269 luminosity changed of a factor of 2, while there are not variations of the spectral shape: the electron temperature $k T_{e}$ is $\sim 23-30 \mathrm{keV}$ and optical depth $\tau$ of the plasma is $\sim 1.2-1.5$. This hard $\mathrm{X}$-ray emission could be interpretate in the standard way, as produced by the upscattering of soft seed photons by a hot, optically thin electron plasma. During the decrease of 
the flux the source show a different spectral shape with a optical depth $\tau$ of the plasma lower than 0.8 and a very high Comptonization plasma temperatures of $k T_{e}$ is $\sim 69-87 \mathrm{keV}$. These measured parameters agree well with the ones from previous BeppoSAX observations (Natalucci et al. 2000), showing the same X-ray spectral behavior: during the decay phase of outbursts (1998 and 2007) no high energy spectral steepening was observed. In this spectral state, spectra are well fitted with a simple power law model, indicating the presence of a possible non thermal electron distribution of velocities.

\section{Ultra Compact X-ray Binary Bursters}

Ultracompact X-ray binaries (UCXBs) are systems with orbital periods $\left(P_{\text {orb }}\right)$ shorter than $\approx 1 \mathrm{hr}$ in which a neutron star or black hole accrete matter from a companion low mass star. We carried out a systematic analysis of all Bursters UCXBs reported in the latest INTEGRAL/IBIS survey (Bird et al. 2007) having the following properties:

a) they are persistent UCXB bursters

b) there are available data in the soft X-ray energy band (at least $\sim 2-8 \mathrm{keV}$ ) from $S W I F T / \mathrm{XRT}$ or BeppoSAX archives.

Details on data reduction and spectral fit results can be found in Fiocchi et al. 2008. The IBIS long monitoring results indicate that UCXB sources spend most of the time in the canonical low/hard state, with the only exception of the source $4 \mathrm{U} 1820$-30 which was always detected in a soft/high state. The luminosities are lower than $\lesssim 7 \times 10^{36} \mathrm{erg} \mathrm{s}^{-1}, k T e$ are typically $\gtrsim 20 \mathrm{keV}$ and $\tau \lesssim 4-5$, but for the source 4U 1820-30 (see Fiocchi et al. 2008, for details).

Figure 6 clearly shows the majority of the sources are in the hard state with constant emission between 10 and $70 \mathrm{keV}$.

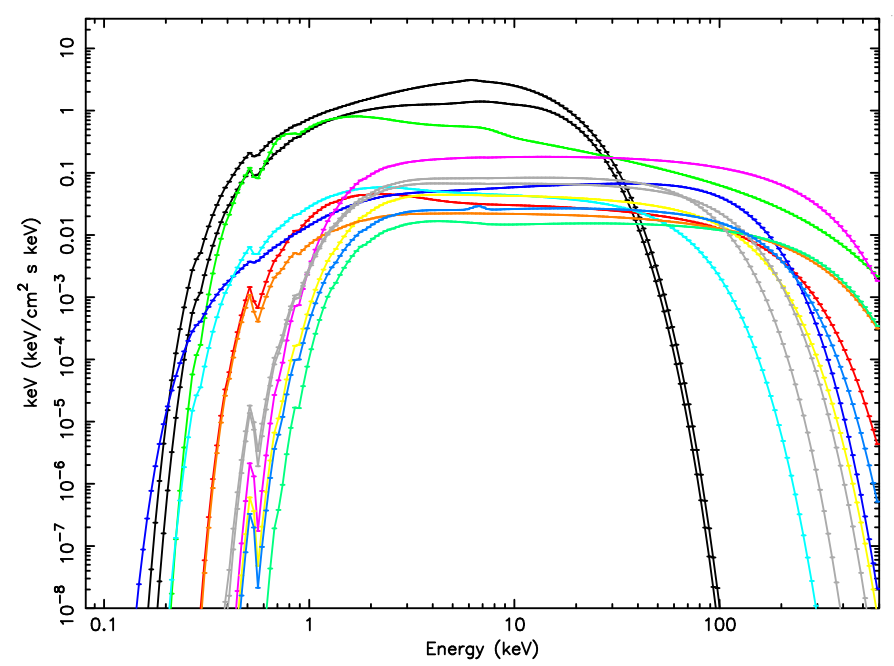

4U 1820-303

4U1850-087

4U 0614+091

XB 1832-330

1 A $1246-588$

4U 1812-12

SAX J1712.

1RXS J172525.5-32

SLX 1735-269

SLX 1737-282

Figure 6: Comparison of the extrapolated models for the burster UCXB observations. 


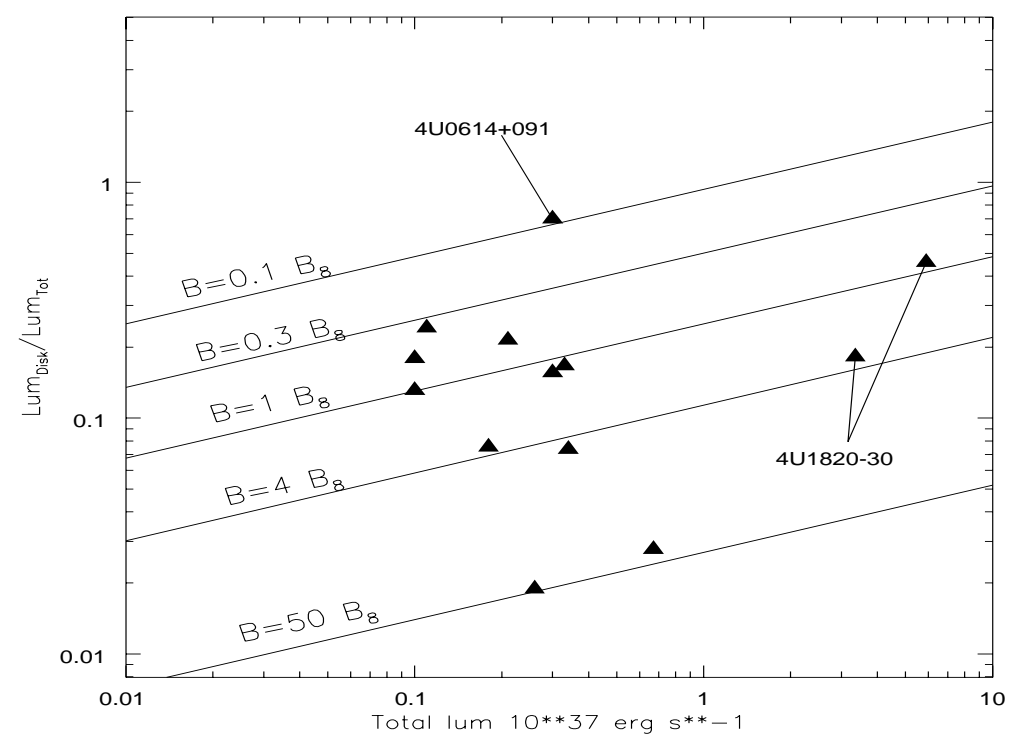

Figure 7: Ratio of the disk to total luminosities versus the total luminosities. Lines are loci of constant magnetic field $\left(0.1,0.3,1,4\right.$, and $50 \times 10^{8} \mathrm{G}$, increasing from top to bottom), assuming a $2 \mathrm{M}_{\odot}$ neutron star and a radius of $10 \mathrm{~km}$.

On the contrary 4U 1820-30 and 4U0614+091 have shown a different energy spectrum shape. The first one is observed only in a typical high/soft state with an accretion rate of $(0.6-1.0) \times$ $10^{-8} \mathrm{M}_{\odot} / y r$, a low electron temperature $(\sim 3 \mathrm{keV})$ and a very high optical depth $(\sim 15-16)$. This source could be a triple system consisting of a white dwarf accreting onto a neutron star and a third body with mass weakly constrained $\left(0.004 \mathrm{M}_{\odot}<M<0.5 \mathrm{M}_{\odot}\right)$, with two timing modulations, the orbital period of $685 \mathrm{~s}$ and super orbital variability of $\sim 170 \mathrm{~d}$ (Zdziarski et al. 2007a, 2007b). The second one, 4U 0614+091, shows a very high electrons temperature $(\sim 140 \mathrm{keV})$. The different spectral shape could be due to non thermal emission nature. Indeed Piraino et al. (1999) interpreted the hard emission as inverse Compton of seed photons with a not Maxwellian electrons distribution.

Assuming the soft component arising from the inner disk, it is possible to estimate the strength of the neutron star magnetic field. The total luminosity can be expressed as $L_{\text {tot }}=G_{n s} \dot{M} / R_{n s}$ and the accretion disk $L_{\text {disk }}$ as $L_{\text {disk }}=G_{n s} \dot{M} / 2 R_{m}$ (Priedhorsky 1986). $R_{m}$ is the magnetospheric radius $\mathrm{R}_{\mathrm{m}}=20 \mathrm{~B}_{8}^{4 / 7} \mathrm{R}_{6}^{10 / 7} \mathrm{M}_{2}^{1 / 7} \mathrm{~L}_{37}^{-2 / 7} \xi \mathrm{km}$

where $B=B_{8} 10^{8} G$ is the magnetic field, $R_{n s}=R_{6} 10 \mathrm{~km}, M_{n s}=2 M_{2} M_{\odot}$ is the neutron star mass, $\mathrm{L}_{\text {tot }}=10^{37} \mathrm{~L}_{37} \mathrm{erg} \mathrm{s}^{-1}$ and the parameter $\xi$ accounts for the disk geometry of the accretion and is $\xi \sim 0.5-1$, (Ghosh \& Lamb 1979, 1992). This formulation allows us to estimate the magnetic field strength of a neutron star from the luminosity of the disk. In Figure 7 a grid with different neutron star magnetic fields, assuming $\mathrm{R}_{6}=1, \mathrm{M}_{2}=1$ and $\xi=1$, is superimposed on a plot of the ratio of disk to total luminosity

$$
\frac{L_{\text {disk }}}{L_{\text {tot }}}=0.25 \mathrm{~B}_{8}^{-4 / 7} \mathrm{R}_{6}^{-3 / 7} \mathrm{M}_{2}^{-1 / 7} \mathrm{~L}_{37}^{2 / 7} \xi
$$

versus the total luminosity $(0.1-100 \mathrm{keV})$. This ratio depends only on the magnetic field strength and total luminosity. The magnetic field estimated in this way for the source $4 \mathrm{U} 1820$ $30, \mathrm{~B}=(0.8-4) \times 10^{8} \mathrm{G}$, is in agreement with the previously derived value $\left(\mathrm{B}=2.1 \times 10^{8} \mathrm{G}\right.$, Sidoli 
et al. 2001; $B=(0.8-9) \times 10^{8} \mathrm{G}$, Campana 2000). Most of the sources presented here show magnetic field in the range $\sim(0.1-5) \times 10^{8} G$. Only 4 U1812-12 and XB1832-33 are characterized by a higher value for the magnetic field.

\section{Conclusions}

Broad band observations have been shown to be a powerful tool to study the accretion flows around neutron stars. Significant progress have been possible recently thanks to INTEGRAL which has both broad band coverage and high sensitivity in hard X-ray. IBIS instrument observed $43 \%$ of LMXBs known. By comparing with the previous editions, this number is costant and represent the $\sim 20 \%$ of $20-100 \mathrm{keV}$ sources and $\sim 45 \%$ of high energy $(>100 \mathrm{keV})$ sources.

Moreover, INTEGRAL observed $~ 50 \%$ of known Bursters and half of these is emitter beyond 100 $\mathrm{keV}$.

The INTEGRAL long monitoring allowed us to study the hard X-ray/Gamma emission of persistent and transient bursters in different spectral state, to discern the physical process in this class of objects. As for BHC, the accretion in hard spectral state of LMXB with neutron star could occur in the form of a truncated accretion disk and a hot inner flow. At higher accretion rate, the inner accretion flow would cool down and collapse, with the disk getting closer to the neutron star. The spectral transitions are associated with accretion rate variations, which changes in turn the temperature of the Comptonizing electrons and the strength of Compton reflection. These can be explained in a model with a truncated outer disk surrounding a hot inner flow, being the truncation radius determinated by the accretion rate.

INTEGRAL data have shown new example of LMXBs with non thermal spectra. This evidence gives us an indication that the mass outflows are important in binary systems with neutron stars because of the possible association with a jet formation.

Finally, UCXB Bursters observed with INTEGRAL showed some common properties:

a) they spend most of time in a typically low luminosity hard state, with X-ray spectra extended up to high energies $(\sim 200 \mathrm{keV})$

b) they are weak persistent sources $\left.\left(\mathrm{L} \sim 10^{36} \mathrm{ergs}^{-1}\right)\right)$

with low mass accretion rate $\left(\sim 10^{-10} M_{\text {solar }} y r^{-1}\right)$

c) they show flat spectra as plotted in $E^{2} F(E)$ from 3 up to $80 \mathrm{keV}$

\section{Acknowledgments}

We acknowledge the ASI financial/programmatic support via contracts ASI-IR I/008/07/0 and I/088/06/0. As one of the thousands Italian researchers with a long-term temporary position, MDS acknowledges the support of Nature $(455,835-836)$ and thanks the Editors for increasing the international awareness of the current critical situation of the Italian Research.

\section{References}

[1] Asai, K., Dotani, T., Mitsuda, K., Inone, H., Tanaka, Y., Lewin, W. H. G., 1998, IAUS, 188, 354 
[ba06] Bazzano et al. 2006, ApJ, 649L, 9

[2] Bird A. J., et al., 2007, ApJ S, 170, 175

[3] Campana S., 2000, ApJ 534, L79

[4] Coppi P. S., 1999, in ASP Conf. Ser. 161, High Energy Processes in Accreting Black Holes, ed. J. Poutanen \& R. Svensson (San Francisco: ASP), 375

[5] D’Amico, F., Heindl, W.A., Rothschild, R.E., Gruber, D.E., 2001, ApJ, 547, 147

[6] Di Salvo et al, 2000, ApJ, 544, 119

[7] Di Salvo, T., Done, C., Życki, P. T., Burderi, L., \& Robba, N. R. 2001, ApJ, 547, 1024

[8] Fiocchi et al. 2006, ApJ, 651, 416

[9] Fiocchi et al. 2007, ApJ, 657, 448

[10] Fiocchi et al. 2008, A\&A, 492, 557

[11] Fiocchi et al. 2009, ApJ, 693, 333

[12] Ghosh P., Lamb F.K., 1979, ApJ 234, 296

[13] Ghosh P., Lamb F.K., 1992, in van den Heuvel E.P.J., Rappaport S.A. (eds.) "X-ray Binaries and Recycled Pulsars", (Dordrecht: Kluwer), p. 487

[14] Iaria, R., Di Salvo, T., Burderi, L., Robba, N. R., 2001, ApJ, 548, 883

[15] Iaria, R., Di Salvo, T., Robba, N. R., Burderi, L., 2002, ApJ, 567, 503

[16] Liu, Q. Z., van Paradijs, J., \& van den Heuvel, E. P. J. 2001, A\&A, 368, 1021

[17] Liu, Q. Z.; van Paradijs, J.; van den Heuvel, E. P. J., 2007, A\&A, 469, 807

[18] Migliari, S., Fender, R. P., Rupen, M., Jonker, P. G., Klein-Wolt, M., Hjellming, R. M., van der Klis, M., 2003, MNRAS, 342, 67

[19] Natalucci L., Bazzano A., Cocchi M., Ubertini P., Heise J., Kuulkers E., In't Zand J.J.M. an d Smith M.J.S., 2000, ApJ, 536, 891

[20] Piraino, S., Santangelo, A., Ford, E. C., Kaaret, P., 1999, A\&A, 349, 77

[21] Priedhorsky W., 1986, ApJ 306, L97

[22] Sidoli, L. et al., 2001, A\&A, 368, 451

[23] Ubertini P., et al., 1998, IAU circ. 6838

[24] Zdziarski A. A., GierliÃĚâĂđski M., Wen L. 2007a, MNRAS, 377, 1017

[25] Zdziarski A. A., Wen L., GierliÃĚÊĂđđski M. 2007b, MNRAS, 377, 1006

[26] Zhang, S. N., et al., 1996, A\&A, 120, 279 